\section{(6) OPEN ACCESS}

\title{
Sex differences in impact of coronary artery calcification to predict coronary artery disease
}

\author{
Yoko M Nakao, ${ }^{1}$ Yoshihiro Miyamoto, ${ }^{1}$ Masahiro Higashi, ${ }^{2}$ Teruo Noguchi, ${ }^{3}$ \\ Mitsuru Ohishi, ${ }^{4}$ Isao Kubota, ${ }^{5}$ Hiroyuki Tsutsui, ${ }^{6}$ Tomohiro Kawasaki, \\ Yutaka Furukawa, ${ }^{8}$ Michihiro Yoshimura, ${ }^{9}$ Hideaki Morita, ${ }^{10}$ Kunihiro Nishimura, ${ }^{11}$ \\ Akiko Kada, ${ }^{12}$ Yoichi Goto, ${ }^{3}$ Tomonori Okamura, ${ }^{13}$ Chuwa Tei, $^{14}$ Hitonobu Tomoike, ${ }^{15}$ \\ Hiroaki Naito, ${ }^{16}$ Satoshi Yasuda ${ }^{3}$
}

\begin{abstract}
- Additional material is published online only. To view please visit the journal online ( http://dx.doi.org/10.1136/ heartjnl-2017-312151).
\end{abstract}

For numbered affiliations see end of article.

\section{Correspondence to} Dr Satoshi Yasuda, National Cerebral and Cardiovascular Center, Osaka, 565-8565, Japan; yasuda.satoshi.hp@ncvc. go.jp

Received 13 July 2017 Revised 17 November 2017 Accepted 28 November 2017 Published Online First 13 January 2018

\section{Check for update}

To cite: Nakao YM, Miyamoto $\mathrm{Y}$, Higashi M, et al. Heart 2018;104:1118-1124.

\section{ABSTRACT}

Objective To assess sex-specific differences regarding use of conventional risks and coronary artery calcification (CAC) to detect coronary artery disease (CAD) using coronary CT angiography (CCTA).

Methods The Nationwide Gender-specific Atherosclerosis Determinants Estimation and Ischemic Cardiovascular Disease Prospective Cohort study is a prospective, multicentre, nationwide cohort study. Candidates with suspected CAD aged $50-74$ years enrolled from 2008 to 2012. The outcome was obstructive CAD defined as any stenosis $\geq 50 \%$ by CCTA. We constructed logistic regression models for obstructive CAD adjusted for conventional risks (clinical model) and CAC score. Improvement in discrimination beyond risks was assessed by C-statistic; net reclassification index (NRI) for CAD probability of low $(<30 \%)$, intermediate $(30 \%-60 \%)$ and high ( $\geq 60 \%)$; and risk stratification capacity.

Results Among 991 patients (456 women, 535 men; 65.2 vs 64.4 years old), women had lower CAC scores (median, 4 vs 60) and lower CAD prevalence (21.7\% vs $37.0 \%$ ) than men. CAC significantly improved model discrimination compared with clinical model in both sexes (0.66-0.79 in women vs $0.61-0.83$ in men). The $\mathrm{NRI}$ for women was 0.33 , which was much lower than that for men (0.71). Adding CAC to clinical model had a larger benefit in terms of moving an additional $43.3 \%$ of men to the most determinant categories (high or low risk) compared with $-1.4 \%$ of women.

Conclusions The addition of CAC to a prediction model based on conventional variables significantly improved the classification of risk in suspected patients with $C A D$, with sex differences influencing the predictive ability. Trial registration number UMIN-CTR Clinical Trial: UMIN000001577.

\section{INTRODUCTION}

In recent decades, there is growing public awareness that cardiovascular disease is an important disease in women and that evidence for sex-specific optimal diagnostic evaluation and management strategies is needed in patients suspected of having coronary artery disease (CAD). ${ }^{1}$ Compared with men, women more often report rare symptoms, ${ }^{3}$ thus making it difficult to evaluate symptoms and precisely predict the likelihood of CAD in women. ${ }^{4}$
In addition, undertesting and undertreatment for CAD lead to higher case fatality rates and increase morbid complications among women. ${ }^{2}$ This is partly because pathophysiology of atherosclerosis differs between sexes. ${ }^{5}$ Thus, considering sex-specific data is important when designing an effective diagnostic method and evaluating the potential uses of any cardiac test. ${ }^{6}$

The measurement of coronary artery calcification (CAC) is a direct marker of the burden of atherosclerosis. ${ }^{7}$ Among symptomatic patients, CAC generally has a high sensitivity and negative predictive value for excluding significant CAD and subsequent adverse cardiovascular events when performed in low-risk and intermediate-risk patients. $^{8}{ }^{9}$ Although there are previous studies about associations between CAC and CAD, and the prognostic impact of CAC for cardiovascular outcomes, the sex-specific impact of CAC for CAD diagnosis is unclear. ${ }^{9-11}$

Our aim was to assess sex differences and the diagnostic value of CAC for CAD in a large, multicentre, prospective cohort study of suspected CAD.

\section{METHODS}

The Nationwide Gender-specific Atherosclerosis Determinants Estimation and Ischemic Cardiovascular Disease Prospective Cohort (NADESICO) study is a prospective, multicentre, cohort study. All patients were provided with written information on the purpose and methods of the study, and they signed a written consent form before participation.

\section{Setting and participants}

Patients were recruited from cardiology departments of participating centres. Baseline recruitment was conducted between December 2008 and April 2013 among 15 hospitals in Japan (online supplementary figure 1). Participants with suspected CAD in a stable setting, who had appropriate indications for plain CT and coronary CT angiography (CCTA), aged 50-74 years and without a history of myocardial infarction or coronary artery revascularisation, were eligible (online supplementary appendix 1). Following the exclusion of seven patients because six patients were excluded with missing data and it was difficult to assess obstructive CAD by CCTA in 


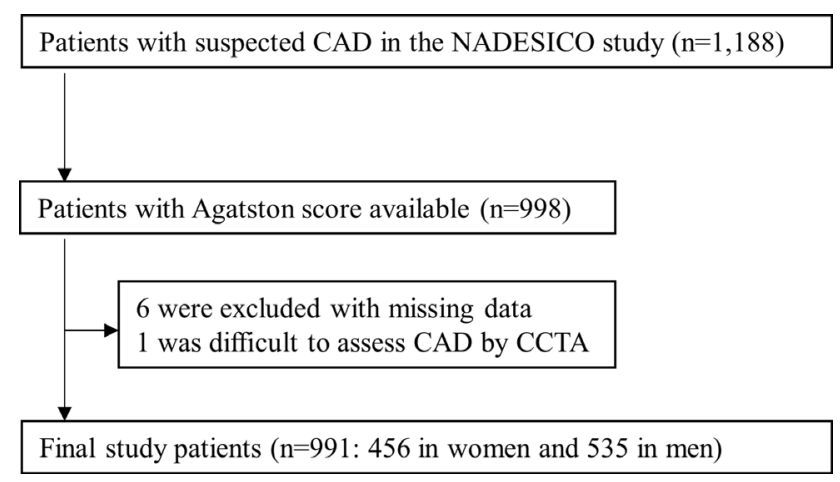

Figure 1 Study flow chart. CAD, coronary artery disease; CCTA, coronary CT angiography; NADESICO, Nationwide Gender-specific Atherosclerosis Determinants Estimation and Ischemic Cardiovascular Disease Prospective Cohort study.

one due to circumferential severe calcification, we finally studied 991 patients (figure 1 ).

\section{Data collection and measurements}

The following clinical data of diagnostic and therapeutic measures were collected by local investigators in the case report forms and sent to the data centre at the National Cerebral and Cardiovascular Center. Data on smoking habit, medical conditions and reproductive history were collected through a self-reported questionnaire at baseline. Laboratory measurements included complete blood count, lipid profiles and levels of fasting plasma glucose.

CT was performed for all patients using 64 or more channels with electrocardiography (ECG) gating according to the established guideline from the Japanese Circulation Society and institutional protocols. The images in Digital Imaging and Communications in Medicine format were transferred to the National Cerebral and Cardiovascular Center and interpreted in a blinded fashion by an independent core laboratory. A single radiologist with extensive experience in coronary CT blinded to all clinical data interpreted the scans with the CAC score, using the Agatston method.

\section{Outcomes}

The outcome was obstructive CAD, defined as one or more vessels with at least 50\% luminal diameter stenosis found using CCTA.

\section{Statistical analysis}

We randomly allocated two-thirds of patients to the derivation dataset and the remaining third to the validation dataset (online supplementary table 1). We used logistic regression model in the derivation dataset to calculate the predicted probability based on standard Framingham risk factors (age, systolic blood pressure, use of blood pressure-lowering medication, total cholesterol, high-density lipoprotein cholesterol and current smoker) stratified by sex (clinical model). ${ }^{12}$ To assess the incremental diagnostic value of the CAC score with respect to conventional risk factors, we added log-transformed CAC score (CAC model). We also analysed results for CAC at clinically relevant thresholds: $0,>0, \geq 100$ or $\geq 400 .{ }^{13}$ We applied the risk equations obtained from the derivation cohort to the validation cohort and calculated measures of discrimination using $\mathrm{C}$-statistic (online supplementary table 2). We compared the clinical model with the CAC model by using C-statistic and net reclassification improvement (NRI). ${ }^{14}$ The NRI estimates were based on the reclassification tables classifying participants into the probability of CAD categories of low $(<30 \%)$, intermediate $(30 \%-$ $<60 \%)$ and high $(\geq 60 \%)^{15}$ using a STATA command 'incrisk'. We plotted the probability of CAD predicted by the CAC model (conventional risks and $\mathrm{CAC}$ ) against the probability of $\mathrm{CAD}$ predicted by the model that included only the conventional risks (clinical model). ${ }^{14}$ Finally, we evaluated the risk stratification capacity that measures a model's capacity to allocate participants from intermediate-risk categories to the highest and lowest risk categories. ${ }^{16}$

We did a sensitivity analysis using altering clinical model ${ }^{41718}$ by selecting references from the recently updated guidelines (online supplementary appendix 2). ${ }^{6}$ As we expected existing models to perform suboptimally in our population with the C-statistics between 0.58 and 0.66 (online supplementary table 4), ${ }^{10} 1920$ we further updated those models (online supplementary table 5). In sensitivity analyses, we used a multivariable random-effect logistic regression model with a random intercept to allow for heterogeneity in CAD prevalence across hospitals.

All analyses were performed using STATA V.13.1.

\section{RESULTS}

\section{Study population}

The study included 991 patients (456 women and 535 men). Table 1 shows characteristics of both sexes of the study population. Women were older than men $(65.2$ vs 64.4 years, $\mathrm{P}=0.047)$ and had lower CAC scores than men (median CAC score, 4 vs $60, \mathrm{P}<0.001)$. Nearly half of women had a CAC score of 0 , and a severe CAC score of $\geq 400$ was less frequent (6.1\%). In men, a CAC score $>0$ to $<100$ was most prevalent (33.6\%), and the other scores were similarly prevalent.

\section{Outcomes}

We observed 297 patients with obstructive CAD, and men had a higher prevalence of CAD $(21.7 \%$ vs $37.0 \%, \mathrm{P}<0.001)$. Figure 2 shows frequencies of each CAC category stratified by the presence of CAD and sex. Women with CAD were evenly divided between those with CAC scores $\angle 100$ and those with CAC scores $\geq 100$. However, half of men with CAD had severe CAC $(\geq 400)$, and only one-quarter of those had CAC scores $<100$. A score of 0 could not exclude CAD in both sexes, especially women with CAD had a higher prevalence of a score of 0 than men $(13.1 \%$ vs $3.0 \%)$.

\section{Prediction of obstructive CAD by CAC}

CAC was significantly associated with CAD in both sexes. The adjusted OR for obstructive CAD was 1.59 (95\% CI 1.35 to 1.86; $\mathrm{P}<0.001)$ in women and 1.77 (95\% CI 1.55 to $2.04 ; \mathrm{P}<0.001)$ in men (table 2). There was also a significant increased risk for CAD between those with a CAC score of 0 and categorical CAC (OR for CAC score of $>0$ to $<100,100$ to $<400$ and $\geq 400$; $3.52,10.74$ and 18.11 in women; 8.40, 26.53 and 83.04 in men; table 2).

\section{Discrimination and reclassification of CAD}

After adding $\mathrm{CAC}$ to the clinical model, the $\mathrm{C}$-statistics significantly improved in both women and men (C-statistic for risk factors only in women: 0.66 (95\% CI 0.58 to 0.73 ); C-statistic after the addition of continuous CAC in women: $0.79(95 \% \mathrm{CI}$ 0.73 to 0.85$)$; C-statistic for risk factors only in men: $0.61(95 \%$ CI 0.55 to 0.67$)$; C-statistic after the addition of continuous 


\begin{tabular}{|c|c|c|c|}
\hline Characteristic & Women $(n=456)(n(\%))$ & Men $(n=535)(n(\%))$ & $P$ value \\
\hline Mean (SD) age, years & $65.2(6.5)$ & $64.4(6.6)$ & 0.047 \\
\hline Current smoker & $34(7.5)$ & $99(18.5)$ & $<0.001$ \\
\hline $\begin{array}{l}\text { Current or past } \\
\text { smoker }\end{array}$ & $87(19.1)$ & $432(80.8)$ & $<0.001$ \\
\hline A history of CAD & $19(4.2)$ & $56(10.5)$ & $<0.001$ \\
\hline Hypertension & $185(40.6)$ & $215(40.2)$ & 0.902 \\
\hline Diabetes mellitus & $114(25.0)$ & $171(32.0)$ & 0.016 \\
\hline Dyslipidaemia & $318(69.7)$ & $372(69.5)$ & 0.944 \\
\hline $\begin{array}{l}\text { Mean (SD) body mass } \\
\text { index, } \mathrm{kg} / \mathrm{m}^{2}\end{array}$ & $23.6(3.7)$ & $24.4(3.2)$ & $<0.001$ \\
\hline $\begin{array}{l}\text { Overweight and } \\
\text { obesity* }\end{array}$ & $141(30.9)$ & $204(38.1)$ & 0.018 \\
\hline Oestrogen status $\dagger$ & $23(5.0)$ & - & - \\
\hline $\begin{array}{l}\text { Mean (SD) systolic } \\
\text { blood pressure, mm } \\
\mathrm{Hg}\end{array}$ & $134.6(18.6)$ & $134.7(17.5)$ & 0.935 \\
\hline $\begin{array}{l}\text { Mean (SD) total } \\
\text { cholesterol, mg/dL }\end{array}$ & $211.4(35.1)$ & $193.5(32.7)$ & $<0.001$ \\
\hline $\begin{array}{l}\text { Mean (SD) HDL } \\
\text { cholesterol, mg/dL }\end{array}$ & $60.8(15.1)$ & $52.4(12.6)$ & $<0.001$ \\
\hline \multicolumn{4}{|l|}{ Coronary calcium score } \\
\hline Median (IQR) & $4(0-78)$ & $60(1-302)$ & $<0.001$ \\
\hline $\begin{array}{l}\text { Mean (SD) log } \\
\text { transformed } \neq\end{array}$ & $2.17(2.32)$ & $3.64(2.56)$ & $<0.001$ \\
\hline 0 & $204(44.7)$ & $120(22.4)$ & $<0.001$ \\
\hline$>0$ to $<100$ & $153(33.6)$ & $180(33.6)$ & \\
\hline 100 to $<400$ & $71(15.6)$ & $122(22.8)$ & \\
\hline$\geq 400$ & $28(6.1)$ & $113(21.1)$ & \\
\hline Obstructive CAD & $99(21.7)$ & $198(37.0)$ & $<0.001$ \\
\hline \multicolumn{4}{|c|}{ Obstructive CAD in patients with } \\
\hline$C A C=0$ & $13(6.4)$ & $6(5.0)$ & $<0.001$ \\
\hline$C A C>0$ to $<100$ & $34(22.2)$ & $45(25.0)$ & \\
\hline CAC 100 to $<400$ & $33(46.5)$ & $56(45.9)$ & \\
\hline$C A C \geq 400$ & $19(67.9)$ & $91(80.5)$ & \\
\hline
\end{tabular}

${ }^{*}$ Defined as BMI $25 \mathrm{~kg} / \mathrm{m}^{2}$ or over.

tOestrogen status was considered positive if women were premenopausal or had oral oestrogen replacement therapy, and negative if they were postmenopausal and were not on oestrogen replacement therapy.

$\ddagger$ Natural logarithm of coronary calcium score +1 .

CAC, coronary artery calcification; $C A D$, coronary artery disease; $H D L$, high-density lipoprotein.

CAC in men: 0.83 (95\% CI 0.78 to 0.87$)$; P value for the difference between models: $\mathrm{P}<0.001$ each) (table 3 ).

Figure 3 shows the probability of $\mathrm{CAD}$ predicted by the CAC model that included CAC against the probability of CAD predicted by the clinical model that included only conventional variables. Horizontal and vertical lines were added at $30 \%$ and $60 \%$ to indicate thresholds of the predicted risk at which a treatment strategy may change as recommended in the guideline. Regarding probability predicted by the clinical model, most women ( $\mathrm{n}=239,81.3 \%)$ had a low probability for CAD $(<30 \%)$, whereas 55 women $(18.7 \%)$ had an intermediate probability (30\%-60\%) (figure 3A, online supplementary table 3). Of $367 \mathrm{men}$, the largest category was intermediate $(\mathrm{n}=278,75.7 \%)$, whereas 89 men $(24.3 \%$ ) had a low probability (figure 3B). For women who appear in the sections on the diagonal (grey area), their risk category was not changed by the addition of CAC to the prediction model. For those in sections off the diagonal, their risk category was changed with the addition of CAC to the prediction model (blue or pink outline). Overall, 57 women and

\section{A Women}

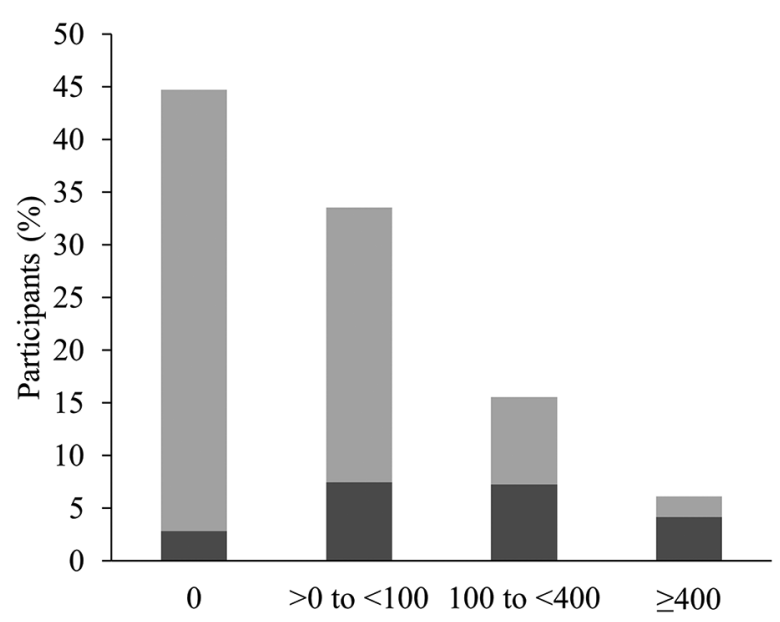

घomen with $\mathrm{CAD} \backsim$ Women without $\mathrm{CAD}$

\section{B Men}

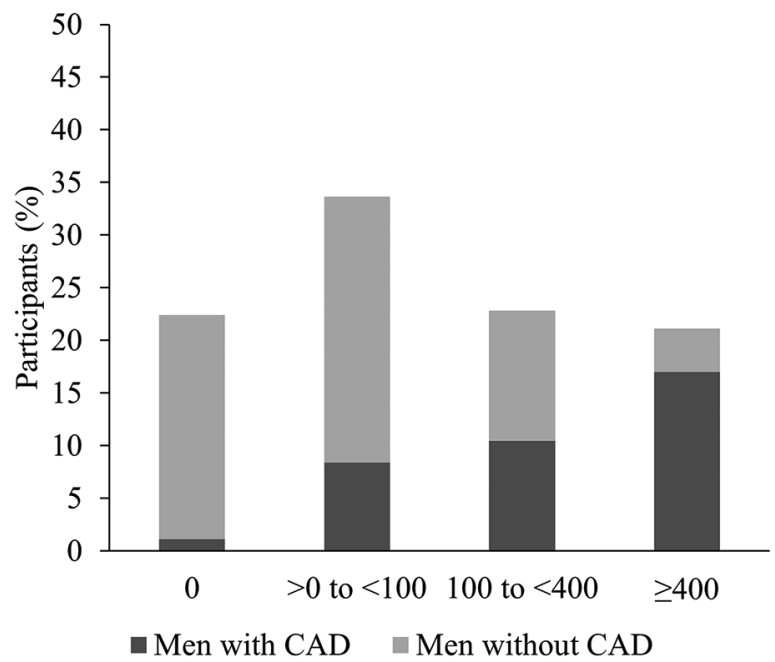

Figure 2 Prevalence of each $C A C$ category stratified with and without $C A D$ by sex: (A) women and (B) men. Dark area=with obstructive $C A D$; light area=without obstructive $C A D$. CAC, coronary artery calcification; $C A D$, coronary artery disease.

112 men were reclassified to a higher risk category (figure 3), with a CAD prevalence of $49.1 \%$ in women and $69.6 \%$ in men. Twenty women and 104 men were reclassified to a lower risk category (figure 3), with a CAD prevalence of $15.0 \%$ in women and $13.5 \%$ in men. The NRI, hence, was 0.33 for women, which was much lower than the value $(0.71)$ for men.

We separately evaluated the clinically meaningful reclassifications, which would presumably have a larger effect on clinical decisions..$^{21}$ After adding the CAC score in the clinical model, 19 women and 87 men were reclassified as having high risk, and 13 (68.4\%) women and 68 (78.2\%) men had CAD.

\section{Risk stratification capacity}

Figure 4 shows the risk stratification capacity of the CAC model. While the clinical model puts $18.7 \%$ of women and $75.7 \%$ of men at intermediate risk, the intermediate-risk values in the CAC model are $20.1 \%$ for women and $32.4 \%$ for men (figure $4 \mathrm{~A}$ ). Therefore, adding CAC to the clinical model has a larger benefit 
Table 2 Logistic regression analysis in the derivation cohort

\begin{tabular}{|c|c|c|c|c|}
\hline & \multicolumn{2}{|l|}{ Women } & \multicolumn{2}{|l|}{ Men } \\
\hline & OR $(95 \% \mathrm{Cl})$ & $P$ value & OR $(95 \% \mathrm{Cl})$ & $P$ value \\
\hline Clinical model ${ }^{*}+$ CAC continuous $t$ & 1.59 (1.35 to 1.86$)$ & $<0.001$ & 1.79 (1.56 to 2.06$)$ & $<0.001$ \\
\hline \multicolumn{5}{|l|}{ Clinical model ${ }^{*}+$ CAC categorical } \\
\hline 0 & Reference & Reference & Reference & Reference \\
\hline$>0$ to $<100$ & 3.52 (1.50 to 8.29$)$ & 0.004 & 8.40 (2.44 to 28.87$)$ & 0.001 \\
\hline 100 to $<400$ & 10.74 (4.01 to 28.81$)$ & $<0.001$ & 26.53 (7.67 to 91.82$)$ & $<0.001$ \\
\hline$\geq 400$ & 18.11 (5.18 to 63.32$)$ & $<0.001$ & 83.04 (22.88 to 301.40 ) & $<0.001$ \\
\hline
\end{tabular}

${ }^{*}$ Clinical model includes age, systolic blood pressure, use of blood pressure-lowering medication, total cholesterol, high-density lipoprotein cholesterol and current smoker. tCAC continuous $=\ln ($ CAC score +1$)$.

CAC, coronary artery calcification.

in terms of moving an additional $43.3 \%$ of men, compared with $-1.4 \%$ of women, to the most determinant, highest and lowest risk categories. With the addition of CAC to the clinical model, an additional $21.0 \%$ of women and $50.0 \%$ of men with CAD were reclassified as having high risk (figure $4 \mathrm{~B}$ ), and an additional $-3.4 \%$ of women and $31.2 \%$ of men without CAD were reclassified as having low risk (figure $4 \mathrm{C}$ ).

\section{Sensitivity analysis}

The sensitivity analysis using different models did not change the overall conclusions (online supplementary tables 5 and 6 and online supplementary figures 2 and 3 ).

\section{DISCUSSION}

The NADESICO study demonstrates that CAC, when added to traditional clinical risks, results in significant improvement in the classification of CAD presence in both women and men. Men achieved a higher NRI and risk stratification capacity than women did, and therefore, appeared to benefit the most from a CAC-based diagnostic strategy. Women, who were reclassified as having high risk, benefited from risk stratification using the CAC model. This study provides strong evidence that there is a significant increase in clinically useful reclassification when CAC is added to risk assessment and addresses an important issue that sex differences should be taken into account.

CAC is part of the development of atherosclerosis and occurs almost exclusively in atherosclerotic arteries; therefore, the CAC score is a direct marker of the burden of atherosclerosis. ${ }^{6} 22$ Previous studies consistently showed that women demonstrated less prevalence and less severity of CAC than men do. ${ }^{2123-26}$ Similarly, in the current series, women had lower CAC scores than men did: scores $>0$ in $55.3 \%$ of women versus $77.6 \%$ of men and $\geq 400$ in only $6.1 \%$ of women versus $21.1 \%$ of men. One recent study in a large population of patients that underwent clinically indicated cardiac catheterisation and optical coherence tomography showed sex differences in plaque microstructure, and women exhibited a lower prevalence of calcification. ${ }^{5}$ These sex differences in the occurrence of coronary calcium and plaque microstructure support the association of CAC with coronary atherosclerosis and underline the importance of sex-specific analyses when evaluating the potential utility of CAC.

The use of measuring CAC in patients suspected as having CAD has been widely studied and discussed in-depth, as seen in the American Heart Association statement. ${ }^{26}$ However, there are limited data broadly specific to women on the relationship between CAC and CAD, and the results are controversial. One previous study with 1764 patients (539 women) with suspected CAD from a single centre showed that there was a significant difference in CAC between men and women, and the reliability of calcium testing in predicting significant stenosis was equally effective in men and women. ${ }^{21}$ Another study with 2115 patients (711 women) with suspected CAD from a single-centre study showed a similar area under the receiver operating characteristic curve stratified by sex, but it did not assess its difference. $^{27}$ Nevertheless, another previous study of 1851 patients (682 women) with suspected CAD demonstrated that although diagnostic performance was similar between women and men, the specificity of the criterion of CAC for detecting obstructive CAD was significantly better in women than in men. ${ }^{23}$ Thus, the sex-specific impact of CAC for CAD diagnosis is unclear.

To assess sex differences in the effects of the addition of new factors on the predictive ability of a model, statistical consideration was needed. The sex-modifiable relationship between $\mathrm{CAC}$ and the development of CAD, including the strength of the base model and the prevalence of CAD in the population under consideration, suggests that the evaluation of model performance may also vary by sex, depending on the measure of predictive ability being evaluated. ${ }^{1}$ In the past three decades, the most commonly used measure to quantify the improvements in prediction models has been the change in the area under the receiver operating characteristic curve. ${ }^{14}$ However, recent studies have emphasised the limitation of the area under the receiver operating characteristic curve, including the difficulty in interpreting the usually small changes in this statistic and the

Table 3 Discrimination and reclassification of CAC for CAD

\begin{tabular}{|c|c|c|c|c|c|c|}
\hline \multirow[b]{2}{*}{ Model } & \multicolumn{3}{|l|}{ Women } & \multicolumn{3}{|l|}{ Men } \\
\hline & C-statistic $(95 \% \mathrm{Cl})$ & NRI & Reclassification (\%) & C-statistic $(95 \% \mathrm{CI})$ & NRI & Reclassification (\%) \\
\hline Clinical model $^{*}$ & $0.66(0.58$ to 0.73$)$ & - & - & $0.61(0.55$ to 0.67$)$ & - & - \\
\hline+ CAC continuoust & 0.79 (0.73 to 0.85$)$ & 0.33 & 24.8 & 0.83 (0.78 to 0.87$)$ & 0.71 & 58.6 \\
\hline+ CAC categorical & $0.78(0.72$ to 0.85$)$ & 0.26 & 22.5 & $0.82(0.78$ to 0.86$)$ & 0.69 & 62.7 \\
\hline
\end{tabular}

*Clinical model includes age, systolic blood pressure, use of blood pressure-lowering medication, total cholesterol, high-density lipoprotein cholesterol and current smoker. †CAC continuous $=$ In (CAC score+1).

CAC, coronary artery calcification; $\mathrm{CAD}$, coronary artery disease; NRI, netreclassification index. 
Figure 3.

(A) Women

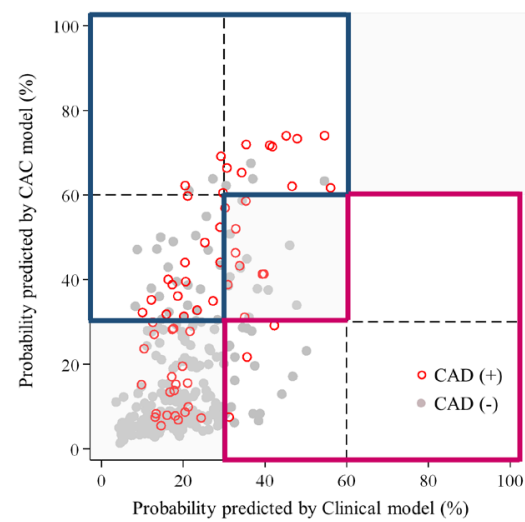

(B) Men

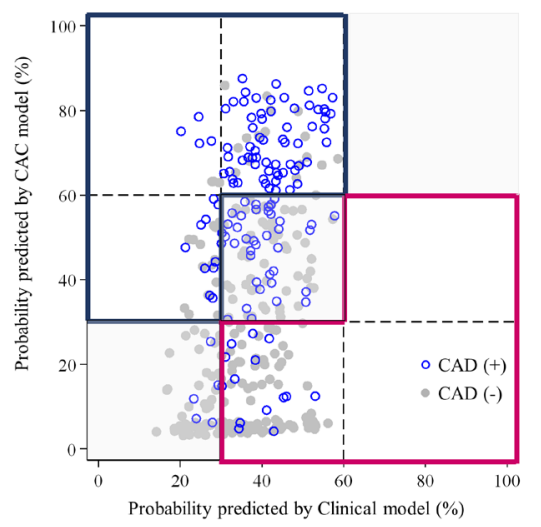

Figure 3 Probability of obstructive CAD predicted by CAC model against the risk predicted by clinical model: $(A)$ women and $(B)$ men. Grey area=both models did not change the risk categories; blue area=reclassified higher categories by CAC model; pink area=reclassified lower categories by $C A C$ model; hollow circle $=C A D$; and solid circle=non-obstructive CAD. The graph shows the probability for $C A D$ in women and men predicted by the clinical model (horizontal axis) against the risk predicted by CAC model (vertical axis). Lines at predicted probabilities of $30 \%$ and $60 \%$ are superimposed to show reclassification over clinically relevant cut points. $C A D$, coronary artery disease; CAC, coronary artery calcification.

relation of the magnitude of improvement to the performance of the baseline model. ${ }^{2829}$ Despite the lower absolute risk of $\mathrm{CAD}$, risk prediction scores, as measured by the area under the receiver operating characteristic curve, have been observed to be higher in women than in men. Therefore, it is necessary to understand sex differences in the epidemiological and statistical determinants of the performance of risk prediction models. ${ }^{1}$ To our knowledge, the current study is the first report of the impact of a CAC-added model on CAD diagnosis using NRI, and we clearly demonstrated sex differences in the CAC-adjusted strategy to diagnose CAD.

Moreover, findings from another metric, that is, risk stratification capacity, showed that the class after reclassification should be carefully interpreted, considering sex difference. In men, adding the CAC score to the clinical model had a demonstrable benefit in terms of moving an additional $43.3 \%$ of patients to the most determinant, highest and lowest risk categories, where diagnostic strategies are better established. However, in women,
Figure 4 .

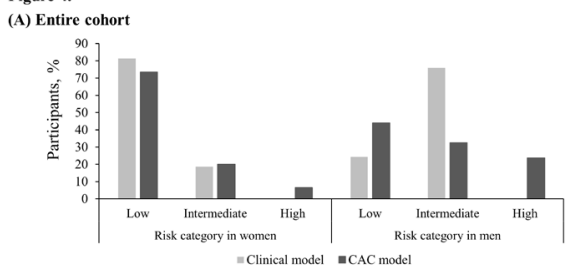

(B) Patients with obstructive $\mathbf{C A D}$

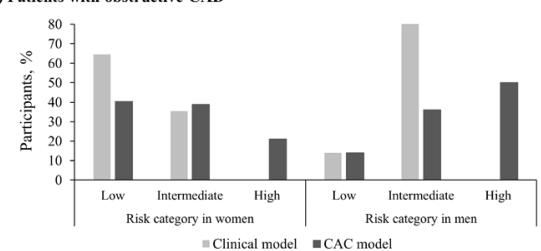

(C) Patients without obstructive CAD

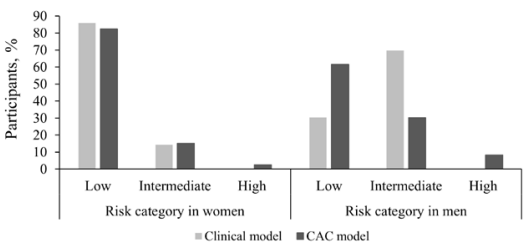

Figure 4 Risk stratification capacity of clinical model and CAC model: (A) entire cohort, (B) patients with obstructive CAD and (C) patients without obstructive CAD. Risk stratification capacity is each model's capacity to allocate participants from intermediate to the highest and lowest risk categories: risk stratification capacity=(the prevalence predicted by clinical model) - (that by CAC model) in figure 4A. $C A C$, coronary artery calcification; $C A D$, coronary artery disease.

the capacity was limited to high-risk patients. Our results demonstrate that although a low-risk category in the CAC model still cannot be used as a marker to diagnose CAD in women because more than $70 \%$ of CAD women was categorised as having low risk in the CAC model, a high-risk category in the CAC model is useful, with the movement of an additional $6.5 \%$ of women to the high category. In patients with $\mathrm{CAD}$, all women and men were not classified as having high risk but as having low-risk or intermediate-risk by the clinical model. Then, adding CAC scores, $21.0 \%$ of women and $50.0 \%$ of men observed with CAD classified as having high risk. The effect of the CAC score for risk prediction is the number of patients identified as having a higher disease risk and, consequently, becoming eligible to receive further evaluation and more intensive therapy because of screening. Moreover, a very important part of the prediction is that there was a sex difference.

Our study has limitations. First, given the cross-sectional design, we were unable to assess the prognostic impact of CAC that would be helpful as a tool to predict future events. We clearly show sex differences of the impact of CAC to help physicians select patients who would benefit from further testing. However, more research into sex difference of prognostic impact of CAC is needed. Second, symptoms for angina are a traditional clinical factor for predicting obstructive CAD, but we cannot sufficiently distinguish atypical angina and non-anginal chest pain because of limited information. No data were available to assess the predictive value of findings based on ECG and other imaging tests. Third, our analysis focused on the diagnostic prediction of the presence of $\mathrm{CAD}$, defined as at least $50 \%$ diameter stenosis in at least one vessel, by CCTA. CCTA can be overdiagnose 
or underdiagnose the obstructive CAD, and this may attenuate the results. Predicting severe stenosis would be a helpful tool to select patients for revascularisation. However, the main purpose of the current analysis was to demonstrate sex differences of CAC to help physicians with further decision making. Fourth, we limited the age group between 50 years and 74 years in the NADESICO study. Women in the reproductive age group will be difficult to participate this study, because CCTA was required for all participants. Other age group that can be changed predictive values should be evaluated in the future research. Moreover, since only $5.0 \%$ of women were oestrogen positive, that is, they were premenopausal or had taken oral oestrogen replacement therapy, we could not assess the effect of oestrogen status. Another limitation maybe overfitting of the model to our data. Although the validation study in the third of our subjects showed similar diagnostic performance characteristics, future studies including external validation of models are needed to fully appreciate the generalisability of the model. Despite these limitations, the study's results suggest that CAC may be a contributory factor to the residual burden of CAD in both men and women. Our results will need to be validated in additional populations.

In conclusion, we found that the CAC score in combination with traditional risk factors enhances the risk classification of CAD in patients with suspected CAD, with sex differences influencing the predictive ability. These results suggest the importance of adopting the sex-specific assessment of CAD.

\section{Author affiliations}

Department of Preventive Medicine and Epidemiologic informatics, National Cerebral and Cardiovascular Center, Suita, Japan

${ }^{2}$ Department of Radiology, National Hospital Organization Osaka National Hospital, Osaka, Japan

\section{Key messages}

\section{What is already known on this subject?}

The measurement of coronary artery calcification is a direct marker of the burden of atherosclerosis. Among patients with suspected coronary artery disease, the coronary artery calcification score generally has a high sensitivity and negative predictive value for excluding significant coronary artery disease and subsequent adverse cardiovascular events when performed in low-risk and intermediate-risk patients.

\section{What might this study add?}

The addition of the coronary artery calcification score to traditional risk factors results in significant improvement in coronary artery disease classification in both sexes. Men exhibited a higher net reclassification index and risk stratification capacity compared with women. They, therefore, appeared to benefit more from a coronary artery calcification-based diagnostic strategy. Women reclassified into the high-risk group benefited more from risk stratification using the coronary artery calcification-added model compared with women in other groups.

\section{How might this impact on clinical practice?}

This study strongly suggests that with the addition of the coronary artery calcification score, a significant increase in clinically useful coronary artery disease reclassification is obtained. Moreover, the study indicates that sex differences are pertinent in coronary artery disease classification.
${ }^{3}$ Department of Cardiovascular Medicine, National Cerebral and Cardiovascular Center, Suita, Japan

${ }^{4}$ Department of Cardiovascular Medicine and Hypertension, Graduate School of Medicine Kagoshima University, Kagoshima, Japan

${ }^{5}$ Department of Cardiology, Pulmonology and Nephrology, Yamagata University School of Medicine, Yamagata, Japan

${ }^{6}$ Department of Cardiovascular Medicine, Graduate School of Medical Sciences, Kyushu University, Fukuoka, Japan

${ }^{7}$ Cardiovascular Center, Shin-Koga Hospital, Kurume, Japan

${ }^{8}$ Department of Cardiovascular Medicine, Kobe City Medical Center General Hospital, Kobe, Japan

${ }^{9}$ Division of Cardiology, Department of Internal Medicine, The Jikei University School of Medicine, Tokyo, Japan

${ }^{10}$ Department of Cardiology, Osaka Medical College, Osaka, Japan

${ }^{11}$ Center for Cerebral and Cardiovascular Disease Information, National Cerebral and Cardiovascular Center, Suita, Japan

${ }^{12} \mathrm{Clinical}$ Research Center, National Hospital Organization Nagoya Medical Center, Nagoya, Japan

${ }^{13}$ Department of Preventive Medicine and Public Health, Keio University, Tokyo, Japan

${ }^{14}$ Waon Therapy Research Institute, Tokyo, Japan

${ }^{15}$ Sakakibara Heart Institute, Fuchu, Japan

${ }^{16}$ Nissay Hospital, Osaka, Japan

Acknowledgements We would like to thank Emiko Terasaka, Toshiko Ishii and Keiko Kuiyama for their support of the study.

Contributors YMN and YM conceived the idea of the manuscript. YMN drafted the first version of the manuscript and led the statistical analysis with support of $\mathrm{KN}$ and $\mathrm{AK}$. YM, MH, TN, YG, HT, HN and SY conceived, designed and supervised the overall study. All authors participated in writing of manuscript, provided important intellectual content and gave their final approval of the version submitted for publication.

Funding This project was supported by grant H20-Junkankitou -Ippan 025 from the Ministry of Health, Labour and Welfare and by Japan Agency for Medical Research and Development under Grant Number JP17gk0210015h0001.

Disclaimer This funding source had no role in the design or implementation of the study or in the writing of this manuscript.

Competing interests None declared.

Ethics approval The study was approved by the Hospital Ethics Committee in the National Cerebral and Cardiovascular Center and regional committee.

Provenance and peer review Not commissioned; externally peer reviewed.

Open access This is an Open Access article distributed in accordance with the Creative Commons Attribution Non Commercial (CC BY-NC 4.0) license, which permits others to distribute, remix, adapt, build upon this work non-commercially, and license their derivative works on different terms, provided the original work is properly cited and the use is non-commercial. See: http://creativecommons.org/ licenses/by-nc/4.0/

(C) Article author(s) (or their employer(s) unless otherwise stated in the text of the article) 2018. All rights reserved. No commercial use is permitted unless otherwise expressly granted.

\section{REFERENCES}

1 Paynter NP, Everett BM, Cook NR. Cardiovascular disease risk prediction in women: is there a role for novel biomarkers? Clin Chem 2014;60:88-97.

2 Mieres JH, Gulati M, Bairey Merz N, et al. Role of noninvasive testing in the clinical evaluation of women with suspected ischemic heart disease: a consensus statement from the American Heart Association. Circulation 2014;130:350-79.

3 Arslanian-Engoren C, Patel A, Fang J, et al. Symptoms of men and women presenting with acute coronary syndromes. Am J Cardiol 2006;98:1177-81.

4 Diamond GA, Forrester JS. Analysis of probability as an aid in the clinical diagnosis of coronary-artery disease. N Engl J Med 1979;300:1350-8.

5 Kataoka Y, Puri R, Hammadah M, et al. Sex differences in nonculprit coronary plaque microstructures on frequency-domain optical coherence tomography in acute coronary syndromes and stable coronary artery disease. Circ Cardiovasc Imaging 2016;9:e004506

6 Greenland P. Bonow RO, Brundage BH, et al. ACCF/AHA 2007 clinical expert consensus document on coronary artery calcium scoring by computed tomography in global cardiovascular risk assessment and in evaluation of patients with chest pain: a report of the American College of Cardiology Foundation Clinical Expert Consensus Task Force (ACCF/AHA Writing Committee to Update the 2000 Expert Consensus Document on Electron Beam Computed Tomography) developed in collaboration with the Society of Atherosclerosis Imaging and Prevention and the Society of Cardiovascular Computed Tomography. J Am Coll Cardiol 2007;49:378-402. 
7 Rumberger JA, Simons DB, Fitzpatrick LA, et al. Coronary artery calcium area by electron-beam computed tomography and coronary atherosclerotic plaque area. A histopathologic correlative study. Circulation 1995;92:2157-62.

8 McBride CB, Cheezum MK, Gore RS, et al. Coronary artery calcium testing in symptomatic patients: An issue of diagnostic efficiency. Curr Cardiovasc Imaging Rep 2013:6:211-20.

9 Skelly AC, Hashimoto R, Buckley DI, et al. Noninvasive testing for coronary artery disease. Rockville 2016.

10 Genders TS, Steyerberg EW, Hunink MG, et al. Prediction model to estimate presence of coronary artery disease: retrospective pooled analysis of existing cohorts. BMJ 2012;344:e3485.

11 Sarwar A, Shaw LJ, Shapiro MD, et al. Diagnostic and prognostic value of absence of coronary artery calcification. JACC Cardiovasc Imaging 2009;2:675-88.

12 Polonsky TS, McClelland RL, Jorgensen NW, et al. Coronary artery calcium score and risk classification for coronary heart disease prediction. JAMA 2010;303:1610-6.

13 Villines TC, Hulten EA, Shaw LJ, et al. Prevalence and severity of coronary artery disease and adverse events among symptomatic patients with coronary artery calcification scores of zero undergoing coronary computed tomography angiography: results from the CONFIRM (Coronary CT Angiography Evaluation for Clinical Outcomes: An International Multicenter) registry. J Am Coll Cardiol 2011;58:2533-40.

14 Leening MJ, Vedder MM, Witteman JC, et al. Net reclassification improvement: computation, interpretation, and controversies: a literature review and clinician's guide. Ann Intern Med 2014;160:122-31.

15 Cooper AC, Skinner N, Sawyer J, et al. Chest pain of recent onset: assessment and diagnosis of recent onset chest pain or discomfort of suspected cardiac origin. London, 2010

16 Janes H, Pepe MS, Gu W. Assessing the value of risk predictions by using risk stratification tables. Ann Intern Med 2008:149:751-60.

17 Morise AP, Haddad WJ, Beckner D. Development and validation of a clinical score to estimate the probability of coronary artery disease in men and women presenting with suspected coronary disease. Am J Med 1997;102:350-6.
18 Pryor DB, Shaw L, McCants CB, et al. Value of the history and physical in identifying patients at increased risk for coronary artery disease. Ann Intern Med 1993;118:81-90.

19 Genders TS, Steyerberg EW, Alkadhi H, et al. A clinical prediction rule for the diagnosis of coronary artery disease: validation, updating, and extension. Eur Heart J 2011;32:1316-30.

20 Moons KG, Donders AR, Steyerberg EW, et al. Penalized maximum likelihood estimation to directly adjust diagnostic and prognostic prediction models for overoptimism: a clinical example. J Clin Epidemiol 2004;57:1262-70.

21 Haberl R, Becker A, Leber A, et al. Correlation of coronary calcification and angiographically documented stenoses in patients with suspected coronary artery disease: results of 1,764 patients. J Am Coll Cardiol 2001;37:451-7.

22 Chandrasekhar J, Mehran R. Sex-based differences in acute coronary syndromes: Insights from invasive and noninvasive coronary technologies. JACC Cardiovasc Imaging 2016;9:451-64.

23 Budoff MJ, Diamond GA, Raggi P, et al. Continuous probabilistic prediction of angiographically significant coronary artery disease using electron beam tomography. Circulation 2002;105:1791-6.

24 Raggi P, Shaw LJ, Berman DS, et al. Gender-based differences in the prognostic value of coronary calcification. J Womens Health 2004;13:273-83.

25 Kay J, Dorbala S, Goyal A, et al. Influence of sex on risk stratification with stress myocardial perfusion Rb-82 positron emission tomography: results from the PET (Positron Emission Tomography) prognosis multicenter registry. J Am Coll Cardiol 2013:62:1866-76.

$26 \mathrm{Kim}$ YJ, Hur J, Lee HJ, et al. Meaning of zero coronary calcium score in symptomatic patients referred for coronary computed tomographic angiography. Eur Heart J Cardiovasc Imaging 2012;13:776-85.

27 Knez A, Becker A, Leber $A$, et al. Relation of coronary calcium scores by electron beam tomography to obstructive disease in 2,115 symptomatic patients. Am J Cardiol 2004;93:1150-2.

28 Cook NR. Use and misuse of the receiver operating characteristic curve in risk prediction. Circulation 2007;115:928-35.

29 Pencina MJ, D'Agostino RB, Pencina KM, et al. Interpreting incremental value of markers added to risk prediction models. Am J Epidemiol 2012;176:473-81. 